\title{
Seesaw tau lepton mass and calculable neutrino masses in a 3-3-1 model
}

\author{
J. C. Montero, ${ }^{*}$ C. A. de S. Pires, ${ }^{\dagger}$ and V. Pleitez \\ Instituto de Física Teórica, Universidade Estadual Paulista, Rua Pamplona, 145, 01405-900, São Paulo, SP, Brazil
}

(Received 14 December 2001; published 17 May 2002)

\begin{abstract}
In this work we show that in a version of the 3-3-1 model proposed by Duong and Ma, in which the introduction of a scalar sextet is avoided by adding a singlet heavy charged lepton, the $\tau$ lepton gains mass through a seesawlike mechanism. We also show how to generate neutrino masses at the one-loop level, and give the respective Maki-Nakagawa-Sakata mixing matrices for a set of the parameters. We also consider the effect of adding a singlet right-handed neutrino.
\end{abstract}

DOI: 10.1103/PhysRevD.65.093017

PACS number(s): 12.15.Ff, 12.60.Cn, 14.60.Fg, 14.60.Pq

Some years ago a model with $\mathrm{SU}(3)_{C} \otimes \mathrm{SU}(3)_{L} \otimes \mathrm{U}(1)_{N}$ gauge symmetry was proposed, 3-3-1 for short, in which the three lepton families transform in the same way under the gauge symmetry, i.e., $\Psi_{a L}=\left(\nu_{a}, l_{a}, l_{a}^{c}\right)_{L} \sim(\mathbf{1}, \mathbf{3}, 0)$ with $a$ $=e, \mu, \tau[1,2]$. Therefore, in this model the lepton mass term transforms as $(1,3,0) \otimes(1,3,0)=\left(1,3^{*}, 0\right)_{A} \oplus(\mathbf{1}, \mathbf{6}, 0)_{S}$; hence, in order to give mass to the charged leptons it is possible to introduce a triplet $\eta=\left(\eta^{0}, \eta_{1}^{-}, \eta_{2}^{+}\right)^{T} \sim(\mathbf{1}, \mathbf{3}, 0)$ and a symmetric sextet $S \sim(\mathbf{1}, \mathbf{6}, 0)$ [2]. With the $\eta$ triplet only, one of the charged leptons remains massless and the other two are mass degenerate. Hence, at least a sextet $S$ has to be introduced in order to give arbitrary masses to all charged leptons. Although this implies that the model has a rather complicated Higgs scalar sector, it is interesting to stress that all the extensions of the electroweak standard model with extra Higgs scalar multiplets, such as multiHiggs doublets [3], singlets [4,5], and triplets [6], are embedded in this 3-3-1 model. In fact, under the subgroup $\mathrm{SU}(2)_{L} \otimes \mathrm{U}(1)_{Y} \subset \mathrm{SU}(3)_{L} \otimes \mathrm{U}(1)_{N}$ the model has three scalar doublets, four singlets (one neutral, one singly charged, and two doubly charged), and a complex triplet. This sort of model also gives some insight into the family replication and into the observed value of the weak mixing angle $\sin ^{2} \theta_{W}$ $<1 / 4$. Moreover, recently the 3-3-1 models have become interesting possibilities for explaining the new value of the positive muon anomalous magnetic moment, reported by the Muon $(g-2)$ Collaboration [7]. The new $(g-2)$ value is consistent not only with supersymmetry [8] but also with several versions of the 3-3-1 model as was shown in Ref. [9]. Finally, in one version of the model it is possible to generate the top and bottom quark masses at the tree level while the other quarks and charged leptons gain mass at the one-loop level [10].

The seesaw mechanism was proposed in order to understand the smallness of the neutrino masses [11]. On the other hand, later, a generalization of this mechanism was introduced, which is also valid for the charged lepton masses, in the context of the minimal left-right symmetric model $[12,13]$. Here we will show, in a 3-3-1 model, that a similar

\footnotetext{
*Email address: montero@ift.unesp.br

†Email address: cpires@ift.unesp.br

₹Email address: vicente@ift.unesp.br
}

mechanism can be implemented for the case of the $\tau$ lepton mass and that the three neutrinos gain mass radiatively. In the case of three neutrinos the masses and mixing are almost completely determined by the charged lepton parameters. In fact, they depend only upon a coupling constant $(\lambda)$ of a quartic term of the scalar potential. This implies that we are also calculating the ratio of the neutrino masses [see Eq. (12) below].

Some years ago, Duong and Ma [14] noted that a way to give mass to the charged leptons that does not involve a scalar sextet can be implemented: In addition to the usual scalar triplets $\eta$, mentioned before, $\rho=\left(\rho^{+}, \rho^{0}, \rho^{++}\right)^{T}$ $\sim(\mathbf{1}, \mathbf{3}, 1)$, and $\chi^{=}\left(\chi^{-}, \chi^{--}, \chi^{0}\right)^{T} \sim(\mathbf{1}, \mathbf{3},-1)$, they introduced a charged lepton transforming like a singlet: $E_{L}^{\prime}$ $\sim(\mathbf{1}, \mathbf{1},-1)$ and $E_{R}^{\prime} \sim(\mathbf{1}, \mathbf{1},-1)$. In this case the Yukawa interactions are given by

$$
\begin{aligned}
-\mathcal{L}_{Y}= & \frac{1}{2} \sum_{\substack{a, b=e, \mu, \tau \\
i, j, k=1,2,3}} \epsilon^{i j k} \overline{\left(\Psi_{a L i}\right)^{c}} F_{a b}(\Psi)_{b L j} \eta_{k} \\
& +\sum_{a=e, \mu, \tau}\left[f_{a} \overline{(\Psi)_{a L}} E_{R}^{\prime} \rho+f_{a}^{\prime} \overline{E_{L}^{\prime}} \chi^{T}\left(\Psi^{c}\right)_{a R}\right] \\
& +M \overline{E^{\prime}}{ }_{L} E_{R}^{\prime}+\text { H.c. },
\end{aligned}
$$

where $F_{a b}$ denotes an antisymmetric matrix. Then the mass matrix for the charged leptons, in the basis of the symmetry eigenstates that we denote $l_{L, R}^{\prime}=\left(e^{\prime}, \mu^{\prime}, \tau^{\prime}, E^{\prime}\right)_{L, R}$, reads $\bar{l}_{L}^{\prime} \mathcal{M}^{l} l_{R}^{\prime}+$ H.c. where

$$
\mathcal{M}^{l}=\frac{1}{\sqrt{2}}\left(\begin{array}{cccc}
0 & -f_{e \mu} v_{\eta} & -f_{e \tau} v_{\eta} & f_{e} v_{\rho} \\
f_{e \mu} v_{\eta} & 0 & -f_{\mu \tau} v_{\eta} & f_{\mu} v_{\rho} \\
f_{e \tau} v_{\eta} & f_{\mu \tau} v_{\eta} & 0 & f_{\tau} v_{\rho} \\
f_{e}^{\prime} v_{\chi} & f_{\mu}^{\prime} v_{\chi} & f_{\tau}^{\prime} v_{\chi} & \sqrt{2} M
\end{array}\right)
$$

Here for simplicity we have assumed real vacuum expectation values: $\left\langle\eta^{0}\right\rangle \equiv v_{\eta} / \sqrt{2},\left\langle\rho^{0}\right\rangle \equiv v_{\rho} / \sqrt{2}$, and $\left\langle\chi^{0}\right\rangle \equiv v_{\chi}^{0} / \sqrt{2}$.

First, by assuming that the only vanishing dimensionless parameters in Eq. (2) are $f_{e \mu}, f_{e \tau}, f_{e}$, and $f_{e}^{\prime}$, we obtain $m_{e}=0$ and approximately 


$$
\begin{aligned}
& m_{\mu} \approx-\frac{f_{\mu} f_{\mu \tau}}{\sqrt{2} f_{\tau}} v_{\eta}, \quad m_{\tau} \approx-\frac{f_{\tau} f_{\tau}^{\prime} v_{\rho} v_{\chi}}{2 M}-m_{\mu}, \\
& m_{E} \approx M-m_{\tau}+m_{\mu} .
\end{aligned}
$$

To get a positive mass for the $\mu$ and $\tau$ leptons we can redefine the corresponding field by a $\gamma_{5}$ factor, i.e., $\mu(\tau)$ $\rightarrow \gamma_{5} \mu(\tau)$ (or see below). We see from Eq. (3) that the $\tau$ lepton mass is of the seesaw type $[12,13]$; however, as shown below, the $M$ mass is not related to a grand unification scale.

The mass matrix in Eq. (2) is diagonalized by a biunitary transformation $U_{L}^{l \dagger} \mathcal{M}^{l} U_{R}^{l}=\hat{\mathcal{M}}^{l}$, relating the symmetry eigenstates (primed fields) with the mass eigenstates (unprimed fields), $l_{L}^{\prime}=U_{L}^{l} l_{L}$ and $l_{R}^{\prime}=U_{R}^{l} l_{R}$, where the mass eigenstates are denoted by $l_{L, R}=(e, \mu, \tau, E)_{L, R}$.

Notice that even for massless neutrinos, which may result in diagonal interactions with the $W$ boson, there are flavor changing neutral and charged currents in the interactions in Eq. (1). This means that although the model has many parameters they are not fixed only by the mass of the charged leptons but by new interactions too. In this situation, we can introduce the unitary matrices $U_{L, R}^{l}$ in Eq. (1) and leave the phenomenology to determine both the masses of the Higgs scalars and the matrix elements of these matrices, or we can determine these matrices first by giving the appropriate mass to the charged leptons and use phenomenology to determine only the mass of the Higgs scalars. Here we will complete the first part of the second alternative, i.e., we will determine the mixing matrices $U_{L, R}^{l}$, and leave for further study the phenomenology of the model.

Since we will be concerned below with the neutrino mass phenomenology, we find it appropriate to obtain a set of parameters that are consistent with the actual values of the charged lepton masses. In this vein we use $U_{L}^{l \dagger} \mathcal{M}^{l}\left(\mathcal{M}^{l}\right)^{\dagger} U_{L}^{l}=\left(\hat{\mathcal{M}}^{l}\right)^{2}$ and $U_{R}^{l \dagger}\left(\mathcal{M}^{l}\right)^{\dagger} \mathcal{M}^{l} U_{R}^{l}=\left(\hat{\mathcal{M}}^{l}\right)^{2}$ in order to find $U_{L, R}^{l}$. The numerical analysis of the eigenvalues of $\mathcal{M}^{l}\left(\mathcal{M}^{l}\right)^{\dagger}\left[\right.$ or $\left.\left(\mathcal{M}^{l}\right)^{\dagger} \mathcal{M}^{l}\right]$, gives the following masses for the charged leptons (in $\mathrm{GeV}$ ):

$$
\begin{aligned}
& m_{e}=0.000510985, m_{\mu}=0.105658, \\
& m_{\tau}=1.77703, m_{E}=3007.79
\end{aligned}
$$

which are in good agreement with those of Ref. [15], if the following parameters are chosen:

$$
\begin{aligned}
& f_{e \mu}=f_{e \tau}=0.0006785, \quad f_{\mu \tau}=0.021, \\
& f_{e}=0.0004263, f_{e}^{\prime}=0.001, \\
& f_{\mu}=0.0481815, f_{\mu}^{\prime}=0.05, \\
& f_{\tau}=0.1259848, f_{\tau}^{\prime}=0.3 ;
\end{aligned}
$$

and also $v_{\chi}=1000 \mathrm{GeV}, M=3000 \mathrm{GeV}, v_{\eta}=20 \mathrm{GeV}$, and $v_{\rho}=245.186\left(=\sqrt{246^{2}-20^{2}}\right) \mathrm{GeV}$. The left- and righthanded mixing matrices, up to five decimal places, are given by

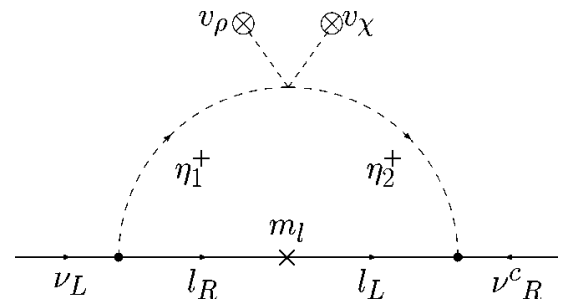

FIG. 1. Diagram showing calculable neutrino masses.

$$
\begin{gathered}
U_{L}^{l} \sim\left(\begin{array}{cccc}
-0.99517 & -0.09776 & 0.00836 & 0.00002 \\
0.08889 & -0.86222 & 0.49866 & 0.00276 \\
-0.04154 & 0.49701 & 0.86672 & 0.00724 \\
0.00008 & -0.00122 & -0.00766 & 0.99997
\end{array}\right), \\
\widetilde{U}_{R}^{l} \sim\left(\begin{array}{cccc}
0.99914 & -0.04130 & 0.00433 & 0.00024 \\
0.04133 & 0.99905 & -0.00754 & 0.01175 \\
0.00395 & -0.00852 & -0.99747 & 0.07053 \\
-0.00100 & -0.01116 & 0.07061 & 0.99744
\end{array}\right) .
\end{gathered}
$$

We have verified that in fact, with the matrices given in Eqs. (6) and (7), $U_{L}^{l \dagger} \mathcal{M}^{l} \widetilde{U}_{R}^{l}=\hat{\mathcal{M}}^{l}$ where $\widetilde{U}_{R}^{l}=U_{R}^{l} \phi$ with $\phi$ $=\operatorname{diag}(1,-1,1,-1)$ and $\hat{\mathcal{M}}^{l}=\operatorname{diag}\left(m_{e}, m_{\mu}, m_{\tau}, m_{E}\right)$. This analysis illustrate how the Duong and Ma singlet lepton works [14] to give the correct mass for the charged leptons.

Until this point the neutrinos remain massless. However, there are several ways to give mass to the neutrinos in the context of the 3-3-1 models. These include mass generation at the tree level [16] or by radiative corrections [17]. Here the neutrinos will continue to be massless unless we allow the breakdown of the $\mathrm{U}(1)_{\mathrm{B}+\mathrm{L}}$ global symmetry in the scalar potential, where $L$ is the total lepton number and $B$ is the baryon number $[18,19]$. This is because, if $B+L$ is conserved, the mixture in the singly charged scalar sector occurs only between $\eta_{1}^{-}, \rho^{-}$, and between $\eta_{2}^{-}, \chi^{-}$, and diagrams like that in Fig. 1 do not occur [see Eq. (8)]. Recall that the $B+L$ assignments are 2 for $\eta_{2}^{-}$and $\chi^{-}$and 0 for $\eta_{1}^{-}$and $\rho^{-}$ [19]; for leptons $B+L$ coincides with $L$ or with the family lepton number. However, if $B+L$ is not conserved a quartic term $\lambda\left(\eta^{\dagger} \chi\right)\left(\eta^{\dagger} \rho\right)+$ H.c. is allowed and all singly charged scalar fields mix with one another. We will consider the case in which this quartic coupling among the scalar Higgs particles does exist and diagrams like that in Fig. 1 do occur. The charged current is written in the mass-eigenstate basis as $\bar{l}_{L} \gamma^{\mu} V_{M N S} \nu_{L} W_{\mu}^{-}$with the Maki-Nakagawa-Sakata matrix [20] defined as $V_{\mathrm{MNS}}=\left(O_{L}^{l}\right)^{\dagger} U_{L}^{\nu}$ where $U_{L}^{\nu}$ is the matrix that relates the symmetry and the left-handed neutrino mass eigenstates. Notice that in this case $V_{M N S}$ is a $4 \times 3$ matrix since $O_{L}^{l}$ is the submatrix $3 \times 4$ of $U_{L}^{l}$, i.e., omitting the fourth row in Eq. (6).

Let us now turn on the lepton interactions with the triplet $\eta$. From Eq. (1) we have

$$
-\overline{l_{\alpha R}}\left(O_{R}^{l T}\right)_{\alpha a} F_{a b} \nu_{b L}^{\prime} \eta_{1}^{-}-\overline{\nu_{a R}^{\prime c}} F_{a b}\left(O_{L}^{l}\right)_{b \alpha} l_{\alpha L} \eta_{2}^{+} .
$$


Notice that in Eq. (8) also only the $3 \times 4$ submatrix of $U_{L, R}^{l}$ appears, i.e., it omits the fourth row in Eqs. (6) and (7), since $a, b=e, \mu, \tau$ but $\alpha=e, \mu, \tau, E$; we have already denoted these submatrices by $O_{L}^{l}, O_{R}^{l}$.

Hence, we have a finite $3 \times 3$ nonsymmetric neutrino mass matrix at the one-loop level of the form

$$
\begin{aligned}
\mathcal{M}_{b d}^{v}= & \lambda v_{\rho} v_{\chi} \sum_{\alpha, a, f}\left(O_{R}^{l T}\right)_{\alpha a} F_{a b} m_{\alpha} F_{d f}\left(O_{L}^{l}\right)_{f \alpha} \\
& \times I\left(M_{1}^{2}, M_{2}^{2}, m_{\alpha}^{2}\right) \\
\approx & \frac{\lambda v_{\rho} v_{\chi}}{16 \pi^{2} M_{2}^{2}} \sum_{\alpha} m_{\alpha}\left(O_{R}^{l T} F\right)_{\alpha b}\left(F O_{L}^{l}\right)_{d \alpha} I\left(r, s_{\alpha},\right),
\end{aligned}
$$

and

$$
I\left(r, s_{\alpha}\right)=\int_{0}^{1} d x \int_{0}^{1-x} d y \frac{1}{\left(s_{\alpha}^{2}-r^{2}\right) x+\left(s_{\alpha}^{2}-1\right) y-s_{\alpha}^{2}},
$$

where $M_{1,2}$ denote typical masses for the singly charged scalars and we have defined $r=M_{1} / M_{2}$ and $s_{\alpha}=m_{\alpha} / M_{2}$. We have neglected a similar contribution proportional to $v_{\eta}^{2}$, which arises from Fig. 1 if we make $\eta_{1}^{-} \rightarrow \rho^{-}, \eta_{2}^{-} \rightarrow \chi^{-}$, and $v_{\rho} v_{\chi} \rightarrow v_{\eta}^{2}$, since we are assuming that $v_{\eta}^{2} \ll v_{\rho} v_{\chi}$.

From Eq. (9) we can built the symmetric mass matrix $M^{\nu}=\left(\mathcal{M}^{\nu}+\mathcal{M}^{\nu T}\right) / 2$. With $M_{1}=100 \mathrm{GeV}, M_{2}=1 \mathrm{TeV}$, and the parameters already obtained for the charged leptons in Eq. (5), we have from Eqs. (9) and (10) numerical values for $M^{\nu}$, in $\mathrm{GeV}$ and up to a factor $10^{-6}$,

$$
M^{\nu} \sim \lambda\left(\begin{array}{ccc}
-0.00775 & -0.18972 & 0.05030 \\
& -4.60084 & 1.2635 \\
& & -0.29346
\end{array}\right) \text {. }
$$

This matrix has the following mass eigenvalues:

$$
\lambda(\approx 0,0.04931,4.95892) \times 10^{-6} \mathrm{GeV},
$$

where the first eigenvalue is assumed to be zero up to the decimal places we are considering.

This time we diagonalize the mass matrix in Eq. (11) by making $\Phi U^{\nu T} M^{\nu} U^{\nu} \Phi=\hat{M}^{\nu}=\operatorname{diag}\left(m_{1}, m_{2}, m_{3}\right)$ with $m_{3}$ $>m_{2}>m_{1}$ where $\Phi=\operatorname{diag}(1,1, i)$ and

$$
U^{\nu} \sim\left(\begin{array}{ccc}
-0.99896 & -0.02268 & 0.03961 \\
0.03228 & 0.26260 & 0.96436 \\
-0.03228 & 0.96464 & -0.26160
\end{array}\right) \text {. }
$$

Notice that the $U^{v}$ matrix does not depend on the global $\lambda$ parameter. In this case we have the mixing matrix $V_{\text {MNS }}$ $\equiv\left(O_{L}^{l}\right)^{T} U^{\nu} \Phi:$

$$
V_{\mathrm{MNS}} \sim\left(\begin{array}{ccc}
0.9983 & 0.0058 & 0.0572 i \\
0.0538 & 0.2552 & -0.9654 i \\
-0.0202 & 0.9668 & 0.2545 i \\
-0.0002 & 0.0077 & 0.0008 i
\end{array}\right),
$$

where we have used the basis $l=(e, \mu, \tau, E)^{T}$ and $\nu$ $=\left(\nu_{1}, \nu_{2}, \nu_{3}\right)^{T}$. Although the first row and column of this mixing matrix are compatible with the small mixing angle (SMA) mixing matrix, we cannot directly compare these numbers with the usual ones [21-23] because the matrix in Eq. (14) is not unitary. This means that, in the case of three neutrinos (three masses), there are more parameters than in the case of a unitary mixing matrix (three Euler angles).

Now we can try to fix the trilinear $\lambda$ parameter to be consistent with the neutrino mass phenomenology. From atmospheric neutrino data [24] we know that at least one mass eigenstate does exist such that its mass is $m>\sqrt{\left|\Delta m_{\text {atm }}^{2}\right|}$ $\sim(4-6) \times 10^{-2} \mathrm{eV}[25]$. On the other hand, the solar neutrino data require $\Delta m_{\odot}^{2} \sim 10^{-5}, 10^{-7}, 10^{-10} \mathrm{eV}^{2}$ depending on the type of solution, large mixing angle (LMA), small mixing angle (SMA), lower probability, low mass (LOW) or vacuum oscillation (VO) solution, respectively [26]. Hence by coherence we will choose $\lambda$ in order to be consistent with the SMA scenario. From Eq. (12) we see that with $\lambda \sim 6.4$ $\times 10^{-5}$ we obtain $m_{1}=0 \mathrm{eV}, m_{2}=3.2 \times 10^{-3} \mathrm{eV}$, and $m_{3}$ $=3.2 \times 10^{-1} \mathrm{eV}$.

Moreover, we would like to stress that the values of the parameters given in Eq. (5) are not expected to be unique. This means that it could be possible to obtain other values in Eqs. (11) and (12) and, of course, of the Maki-NakagawaSakata (MNS) matrix in Eq. (14), which could fit better the experimental data [21]. We have verified also that the neutrino masses and the mixing matrix do not strongly depend on the masses of the charged scalars $M_{1}, M_{2}$. In this way, the neutrino parameters are a prediction of the model: Once the values of the $f$ 's in Eq. (2) are made consistent with the known charged lepton masses, the ratios of the neutrino masses and the mixing matrix $U^{\nu}$ are also automatically fixed, since $\lambda$ is a common factor.

Next, we would like to point out that in the 3-3-1 model the introduction of fermion singlets is more natural than in other models, including the standard electroweak model, because in the present context the charged singlet is introduced in order to replace the scalar sextet. Since the number of fermion families transforming nontrivially under the 3-3-1 gauge symmetry must be a multiple of 3 , the more economic way to introduce new fermions is by adding singlets under this symmetry. Moreover, in the present model which already has a charged lepton singlet, if we want to have again a symmetry in the representation content between the charged leptons and neutrinos we can add a right-handed neutrino singlet $N_{R}^{\prime}$. In this case we can obtain a wider neutrino mass spectrum if we consider $N_{R}^{\prime}$ as a light neutrino. In this situation we have the interactions

$$
\sum_{a=e, \mu, \tau} \hat{h}_{a}^{\prime} \overline{\psi_{a L}} N_{R}^{\prime} \eta+\frac{1}{2} \overline{\left(N_{R}^{\prime}\right)^{c}} M_{R} N_{R}^{\prime}+\text { H.c. . }
$$


The neutrino mass matrix in the basis $N^{\prime}$ $=\left(\nu_{e L}^{\prime}, \nu_{\mu L}^{\prime}, \nu_{\tau L}^{\prime},\left(N^{\prime c}\right)_{L}\right)^{T}$ and up to a $10^{-6}$ factor, in $\mathrm{GeV}$, is given by

$$
M^{\nu} \sim \lambda\left(\begin{array}{cccc}
-0.00775 & -0.18972 & 0.05030 & \frac{\hat{h}_{e} v_{\eta}}{2 \sqrt{2} \lambda} \\
& -4.60838 & 1.2635 & \frac{\hat{h}_{\mu} v_{\eta}}{2 \sqrt{2} \lambda} \\
\frac{\hat{h}_{e} v_{\eta}}{2 \sqrt{2} \lambda} & \frac{\hat{h}_{\mu} v_{\eta}}{2 \sqrt{2} \lambda} & \frac{\hat{h}_{\tau} v_{\eta}}{2 \sqrt{2} \lambda} & \frac{M_{R}}{2 \lambda}
\end{array}\right),
$$

where the numerical $3 \times 3$ submatrix denotes the mass entries generated by radiative corrections already obtained in Eq. (11); we have defined $\hat{h}_{a}=10^{6} \hat{h}_{a}^{\prime}$. The mass matrix in Eq. (16) has the following mass eigenvalues:

$$
(\approx 0,0.005,0.052,1.052) \mathrm{eV}
$$

if we choose the following values for the dimensionless parameters:

$$
\left(\lambda, \hat{h}_{e}, \hat{h}_{\mu}, \hat{h}_{\tau}\right)=\left(10^{-6}, 2 \times 10^{-3}, 10^{-7}, 10^{-7}\right),
$$

and $v_{\eta}=20 \mathrm{GeV}, M_{R}=2 \mathrm{eV}$. We can identify, for instance, $\Delta m_{43}^{2}=1.1 \mathrm{eV}^{2}, \Delta m_{32}^{2}=2.7 \times 10^{-3} \mathrm{eV}^{2}, \quad$ and $\Delta m_{21}^{2}=2.1$ $\times 10^{-5} \mathrm{eV}^{2}$ with the respective mass square difference consistent with Liquid Scintillation Neutrino Defecter (LSND) [27], atmospheric [24], and solar [26] neutrino data. In this case the mixing matrix in the neutrino sector is given by

$$
U^{\nu} \sim\left(\begin{array}{cccc}
0.99912 & -0.04199 & -0.00191 & -0.00067 \\
-0.04193 & -0.99886 & 0.02275 & -0.00091 \\
-0.00293 & -0.02191 & -0.97602 & -0.21654 \\
0 & 0.00582 & 0.21646 & -0.97627
\end{array}\right) \text {. }
$$

The relation between the phenomenological neutrinos $N_{a}^{\prime}$ and the massive ones $N=\left(\nu_{1}, \nu_{2}, \nu_{3}, \nu_{4}\right)^{T}$ is given by $N^{\prime}$
$=U^{\nu} \Phi N$ where $\Phi=\operatorname{diag}(1, i, i, 1)$. The $V_{\mathrm{MNS}}=U_{L}^{l \dagger} U^{\nu} \Phi$ mixing matrix is given now by

$$
V_{\mathrm{MNS}} \sim\left(\begin{array}{cccc}
-0.9979 & -0.0461 i & 0.0445 i & 0.0095 \\
-0.0630 & 0.8544 i & -0.5048 i & -0.1056 \\
-0.0151 & -0.5175 i & -0.8363 i & -0.1807 \\
-0.0001 & 0.0029 i & 0.2094 i & -0.9778
\end{array}\right) .
$$

Notice that the $3 \times 3$ (non-Hermitian) submatrix involving the known charged leptons and the three active neutrinos is again of the SMA solution type of the solar neutrino problem $[21,23]$. However, see the discussion above. In this case we also obtain the mass square differences which are appropriate for the solar and atmospheric neutrino data. From the fourth row and column we see that the heavy charged lepton couples mainly with the fourth neutrino and the usual charged leptons couple weakly to this neutrino. Notwithstanding, in this case also the mixing matrix is not orthogonal and it is not possible to make a direct comparison with the usual $3 \times 3$ fitting of the data; and, as we said before, using a different set of parameters in Eqs. (5) and (18) we should obtain different mixing matrices.

Thus, in this context we have four phenomenological neutrinos denoted by $\nu_{e L}, \nu_{\mu L}, \nu_{\tau L},\left(N^{\prime c}\right)_{L}$ in the symmetry basis. So, in general, we have the possibility of implementing " $2+2$ " [28], "3+1" [29-31], or intermediate neutrino mixing schemes. However, it is worth recalling that it is not clear yet that we need four neutrinos since other types of solutions like flavor changing neutrino interactions, are still possible for the solar neutrino [32,33]. Also, for the case of the atmospheric neutrino, more exotic solutions like neutrino decay and decoherence are not ruled out yet [34]. Moreover, several of these mechanisms can be working at the same time. Notice also that without the sextet we have no chance to assume that the charged lepton mass matrix is diagonal, and that the fourth row gives the couplings of the neutrinos with the heavy charged lepton, which are very small.

Finally, we would like to recall that in the present model there are energy independent flavor conversions [35].

This work was supported by Fundação de Amparo à Pesquisa do Estado de São Paulo (FAPESP), Conselho Nacional de Ciência e Tecnologia (CNPq), and Programa de Apoio a Núcleos de Excelência (PRONEX).
[1] F. Pisano and V. Pleitez, Phys. Rev. D 46, 410 (1992).

[2] P.H. Frampton, Phys. Rev. Lett. 69, 2889 (1992); R. Foot, O.F. Hernandez, F. Pisano, and V. Pleitez, Phys. Rev. D 47, 4158 (1993).

[3] J.F. Gunion, H.E. Haber, G.L. Kane, and S. Dawson, The Higgs Hunter's Guide (Addison-Wesley, Reading, MA, 1999).

[4] Y. Chikashige, R.N. Mohapatra, and R.D. Peccei, Phys. Lett. 98B, 265 (1981).

[5] A. Zee, Phys. Lett. 93B, 389 (1980).
[6] T.P. Cheng and L.-F. Li, Phys. Rev. D 22, 2860 (1980).

[7] Muon $(g-2)$ Collaboration, H.N. Brown et al., Phys. Rev. Lett. 86, 2227 (2001).

[8] J.L. Lopez, D. Nanopoulos, and X. Wang, Phys. Rev. D 49, 366 (1994); U. Chattopadhyay and P. Nath, ibid. 53, 1648 (1996); T. Mori, ibid. 53, 6565 (1996).

[9] N.A. Ky, H.N. Long, and D. Van Soa, Phys. Lett. B 486, 140 (2000); see also H. Hujii, S. Nakamura, and K. Sasaki, ibid. 299, 342 (1993); C.A. de S. Pires and P.S. Rodrigues da Silva, 
Phys. Rev. D 64, 117701 (2001).

[10] M.D. Tonasse, Nucl. Phys. B623, 316 (2002).

[11] M. Gell-Mann, P. Ramond, and R. Slansky, in Supergravity, edited by P. van Nieuwenhuisen and D.Z. Freedman (NorthHolland, Amsterdam, 1979); T. Yanagida, in Proceedings of the Workshop on the Unified Theory and the Baryon Number in the Universe, edited by O. Sawada and A. Sugamoto (KEK Report No. 79-18, Tsukuba, Japan, 1979); R.N. Mohapatra and G. Senjanovic, Phys. Rev. Lett. 44, 912 (1980).

[12] A. Davidson and K.C. Wali, Phys. Rev. Lett. 59, 393 (1987).

[13] Y.A. Coutinho, J.A. Martins Simões, and C.M. Porto, Eur. Phys. J. C 18, 779 (2001).

[14] T.V. Duong and E. Ma, Phys. Lett. B 316, 307 (1993).

[15] Particle Data Group, D.E. Groom et al., Eur. Phys. J. C 15, 1 (2000).

[16] M.B. Tully and G.C. Joshi, Phys. Rev. D 64, 011301 (2001); J.C. Montero, C.A. de S. Pires, and V. Pleitez, Phys. Lett. B 502, 167 (2001).

[17] P.H. Frampton, P.I. Krastev, and J.T. Liu, Mod. Phys. Lett. A 9, 761 (1994); Y. Okamoto and M. Yasue, Phys. Lett. B 466, 267 (1999); T. Kitabayashi and M. Yasue, Phys. Rev. D 63, 095002 (2001); hep-ph/0006040.

[18] V. Pleitez and M.D. Tonasse, Phys. Rev. D 48, 5274 (1993).

[19] J.C. Montero, C.A. de S. Pires, and V. Pleitez, Phys. Rev. D 60, 115003 (1999).

[20] Z. Maki, M. Nakagawa, and S. Sakata, Prog. Theor. Phys. 28, 246 (1962).

[21] The preferred solution to the solar neutrino problem is the LMA solutions, see A.M. Gago et al., Phys. Rev. D 65, 073012 (2002), and references therein.

[22] M. Fukugita, M. Tanimoto, and T. Yanagida, Phys. Rev. D 57, 4429 (1998).
[23] S.K. Kang, C.S. Kim, and J.D. Kim, Phys. Rev. D 62, 073011 (2000).

[24] SuperKamiokande Collaboration, Y. Fukuda et al., Phys. Rev. Lett. 81, 1562 (1998); 82, 2644 (1999).

[25] A.Yu. Smirnov, talk given at the XIX International Conference on Neutrino Physics and Astrophysics, Neutrinos-2000, Sudbury, Canada, 2000, hep-ph/0010097.

[26] Homestake Collaboration, B.T. Cleveland et al., Astrophys. J. 496, 505 (1998); Kamiokande Collaboration, K.S. Hirata et al., Phys. Rev. Lett. 77, 1683 (1996); GALLEX Collaboration, W. Hampel et al., Phys. Lett. B 477, 127 (1999); SAGE Collaboration, J.N. Abdurashitov et al., Phys. Rev. Lett. 77, 4708 (1996); Phys. Rev. C 60, 055801 (1999); SNO Collaboration, Q.R. Ahmad et al., Phys. Rev. Lett. 87, 071301 (2001).

[27] LSND Collaboration, C. Athanassopoulos et al., Phys. Rev. Lett. 77, 3082 (1996); 81, 1774 (1998).

[28] V. Barger, T.J. Weiler, and K. Whisnant, Phys. Lett. B 427, 97 (1998); S.M. Bilenky, C. Giunti, W. Grimus, and T. Schwetz, Phys. Rev. D 60, 073007 (1999).

[29] V. Barger, B. Kayser, J. Learned, T. Weiler, and K. Whisnant, Phys. Lett. B 489, 345 (2000).

[30] B. Kayser, "Neutrino Mass: The Present and the Future," in Proceedings of the XXXth International Conference on High Energy Physics, Osaka, Japan, 2000, hep-ph/0010206, and references therein.

[31] O.L.G. Peres and A.Y. Smirnov, Nucl. Phys. B612, 59 (2001).

[32] S. Bergmann, M.M. Guzzo, P.C. de Holanda, P.I. Krastev, and H. Nunokawa, Phys. Rev. D 62, 073001 (2000); A. M. Gago et al., ibid. 65, 073012 (2002).

[33] A.M. Gago, E.M. Santos, W.J.C. Teves, and R. Zukanovich Funchal, Phys. Rev. D 63, 073001 (2001).

[34] O. Yasuda, hep-ph/0102167, and references therein.

[35] M.M. Guzzo et al., hep-ph/9908308. 